\title{
Perception of slant-in-depth is automatic
}

\author{
WILLIAM EPSTEIN and TIMOTHY BABLER \\ University of Wisconsin-Madison, Madison, Wisconsin
}

\begin{abstract}
Two experiments assessed perception of the slant-in-depth of static irregularly contoured shapes when attention was withdrawn from processing slant-in-depth. The experiments showed that when the memory load of the experimental task is minimal, discrimination of slant-in-depth is evidenced even when attention has not been directed to slant discrimination. The findings are brought to bear on a model of perceiving shape-at-a-slant (Epstein \& Lovitts, 1985) that partitions the components of the process into automatic and attention-demanding operations.
\end{abstract}

In earlier work, we compared judgments of shape-at-aslant (Epstein \& Lovitts, 1985) and size-at-a-distance (Epstein \& Broota, 1986) under two attentional conditions. In one condition, attention was directed to processing of shape (Epstein \& Lovitts, 1985) or processing of size (Epstein \& Broota, 1986); in the corresponding contrasting condition, attention was withdrawn from processing of shape or size. On a two-alternative forced-choice (2AFC) test, which offered the subject a choice between an objective and a projective match for the previously encountered standards, subjects in the shape-directed and size-directed attentional conditions chose the objective alternative as a match for the standard. In sharp contrast, when attention had been withdrawn from processing of shape or size, subjects chose the projective alternative as a match for the standard. We took these findings as support for a partial automaticity hypothesis. This is the proposal that the process adumbrated by the shape-slant invariance hypothesis and the size-distance invariance hypothesis (Epstein, 1973, 1977; Hochberg, 1978; Rock, 1975, 1983) is constituted of two types of operations: automatic operations that register and represent projective shape (or projective size) and slant-in-depth (or absolute distance), and attentional operations that integrate the outputs of the automatic operations to form object-centered descriptions of shape and size. When attention is withdrawn, only the outputs of the automatic operations are available; hence, the preference is for projective matches on the 2AFC test. When attention is directed to shape and size processing, the output of the full process is available; hence, the preference is for the objective match.

In the earlier work, we did not solicit information concerning perceived slant-in-depth or perceived distance. In the present experiments, we remedied this shortfall for the case of slant-in-depth. There are two reasons for our concern with these data. First, in the presentations of the model (e.g., see Epstein \& Lovitts, 1985, Figure 1), we have assumed that registration of the optical correlates of slant-

This article is based upon work supported by the National Science Foundation under Grant BNS-8706858 awarded to William Epstein. Address correspondence to William Epstein, Department of Psychology, University of Wisconsin-Madison, Madison, WI 53706. in-depth and representation of slant-in-depth is automatic. Evidence from judgments of slant-in-depth is needed to evaluate this assumption.

In addition to complementing the data in hand, the results of the present experiments are important in assessment of a rival account of the obtained findings. A number of studies (Coren \& Porac, 1983; Gogel, 1967; Gogel, Loomis, Newman, \& Sharkey, 1985; Gogel \& Tietz, 1976; Peterson, 1986; Tsal, 1984) suggest that the efficacy of depth cues is affected by allocation of attention. Suppose that, in the experiments by Epstein and Lovitts (1985) on perceived shape-at-a-slant, when attention is diverted from processing of depth cues, depth is assigned a default value. In the case of slant-in-depth, the default assignment is the frontoparallel orientation. (Ample evidence that such tendencies are at work even when depth cues are processed is provided in an extensive series of studies by Gogel, 1967, 1978.) If an object that is rotated in depth is registered as occupying the frontal plane, the shape-slant algorithm will generate a shape that conforms to the projective (viewer-centered) shape. In this case, however, the reported shape is not evidence of the output of automatic operations or of the need for attention to ensure an object-centered description.

Measures of perceived slant-in-depth under varying attentional conditions (attention directed to processing of slant vs. attention diverted from processing of slant) should provide a basis for assessing the alternative interpretation. If the account that we have offered (Epstein \& Lovitts, 1985) is correct, reports of perceived slant-in-depth should be positively correlated with objective slant-in-depth, both when attention is directed to processing slant and when attention is diverted from discriminations of slant. If the rival account is correct, judgments of slant-in-depth should be correlated with objective slant only when attention is directed to discrimination of slant; when attention is diverted from discrimination of slant, perceived slant should be independent of objective slant, reflecting instead the tendency to assign all shapes to the frontoparallel orientation.

The general plan of the experiments involved comparisons of slant-in-depth judgments under two attentional conditions in a situation modeled after Epstein and Lovitts's (1985) experiments on the effect of attention on perceived 
shape. In the slant-directed condition, attention was directed to processing of slant-in-depth. In the numerosity-directed condition, attention was withdrawn from processing of slant-in-depth.

The key comparison is between the slant-directed and numerosity-directed conditions. The comparison can be exploited twice to address the two concerns that motivated the experiments: (1) If representation of slant-in-depth is automatic, performance on the 2AFC test of slant-in-depth under the numerosity-directed condition should be comparable to test performance under the slant-directed condition. In both cases, performance should be significantly above chance levels. (2) If the default hypothesis is correct, performance on the 2AFC test under the numerositydirected condition should be significantly poorer than under the slant-directed condition, owing to a marked tendency to perceive the standards as being in the frontoparallel orientation under the former condition. On the 2AFC slant test, which offers a choice between the rotated-in-depth and frontoparallel alternatives, a significant bias favoring the frontoparallel alternative should be evident under the numerosity-directed condition. It is obvious that the evidence required to satisfy the default hypothesis (i.e., no evidence of a bias for the frontoparallel plane) is less demanding than the evidence required to support the current version of the partial automaticity hypothesis (i.e., evidence that slant-in-depth is discriminated under the numerosity-directed condition).

\section{EXPERIMENT 1}

\section{Method}

Subjects. The subjects were $\mathbf{4 0}$ introductory psychology students at the University of Wisconsin-Madison. All subjects had normal or corrected-to-normal vision.

Stimuli. Eight different eight-point shapes were chosen from Vanderplas and Garvin's (1959) compilation of irregularly contoured shapes. Four of the selected shapes had high association value $(M=42.5)$, and four shapes had low association value $(M=24)$. Two identical sets of the eight shapes were constructed from white poster board and mounted on black stalks. A vertical array of black dots, ranging in number from five to eight, was placed along the vertical axis of each shape. The dots were $3 \mathrm{~mm}$ in diameter and $3 \mathrm{~mm}$ apart. One member of each pair of shapes displayed an odd number of dots, whereas the other member displayed an even number of dots.

Test sheets showed a miniature replica of one of the eight-point shapes, the response alternatives ("rotated in depth" and "not rotated in depth"), and a scale for a confidence rating of the subject's response.

Apparatus. The apparatus consisted of a two-field tachistoscope, a microprocessor that controlled the exposure duration of the tachistoscope fields, and a two-button response panel. Each field in the tachistoscope had a carousel with eight locations. At each location was a stalk bearing one of the eight shapes. The shapes could be positioned either in the frontoparallel plane or rotated in depth at $60^{\circ}$ around the vertical axis. The carousels could be rotated to position the stimulus directly in front of the viewing window. The buttons on the response panel were labeled "odd" and "even."

Procedure. All subjects were initially tested under the numerositydirected condition. The subjects were instructed that their task was to make odd-even discriminations of the number of dots on briefly presented backgrounds. The subjects were instructed to press the button labeled "odd" if there was an odd number of dots and to press the button labeled "even" if there was an even number of dots. A sample shape with three dots was used to illustrate the task. Neither shape nor slant discrimination was mentioned in the description of the task.

After the instructions were administered, two of the eight standard shapes were presented in succession. The exposure duration of each shape was $1 \mathrm{sec}$, with about a 3-sec intershape interval. One of the two shapes was presented in the frontoparallel position; the other shape was presented rotated in depth at $60^{\circ}$ around the vertical axis.

Immediately following presentation of the second shape, test instructions were administered. The subjects were asked to identify the orientation occupied by each shape by choosing between two alternatives: "rotated in depth" and "not rotated in depth." These two orientations were demonstrated using a sample shape. On each page of the test booklet, the subjects identified the orientation occupied by the shape represented by the miniature replica shown at the top of the page. The subjects also rated the confidence of their responses on a scale from just guessing to absolutely certain. The confidence rating was elicited to encourage conscientious performance, but was not analyzed.

Upon completion of this two-item test, the subjects were tested under the slant-directed condition. The subjects were informed that two new shapes would be presented in the manner similar to that of the initial two shapes. The subjects were directed to concern themselves solely with discriminating the shape's orientation. No oddeven response was requested. As in the first part, one shape was presented in the frontoparallel plane and the other shape was rotated in depth at $60^{\circ}$. Immediately following presentation of the second of the new pair of shapes, test instructions were read to the subjects. These test instructions were the same as those used after the initial two trials. The test instructions were repeated to ensure an equal time interval between the last study trial and the shape-orientation test for both conditions.

Across all subjects, each of the eight shapes was presented equally often in the frontoparallel and the rotated-in-depth positions, equally often with odd dots and even dots, equally often first and second, and equally often in the first condition and second condition. The pairing of shapes was randomized with the restriction that each shape appeared equally often across subjects.

\section{Results}

The results of Experiment 1 are summarized in Table 1. Under the numerosity-directed condition, the proportion correct was .762; under the slant-directed condition, the proportion correct was .962 . Performance under each condition was significantly better than chance by the binomial test $(p<.001)$. Odd-even discrimination under the numerosity-directed condition was correct on $68.7 \%$ of the trials. The results provide clear evidence of objectcorrelated representation of slant-in-depth in the absence of focused attention.

\section{EXPERIMENT 2}

Experiment 2 was designed to examine the same question under conditions that reduced the memory demands

Table 1

Proportion Correct Under the Attentional Conditions of Experiment 1

\begin{tabular}{lccc}
\hline Attentional Condition & Frontoparallel & Rotated & Overall \\
\hline Slant-Directed & .950 & .975 & .962 \\
Numerosity-Directed & .775 & .750 & .762 \\
\hline
\end{tabular}


of the test to the barest minimum. Experiment 2 followed the procedure of Experiment 1 except for a revision in the form of the slant test. After presentation of the second form, the sequence of presentations was interrupted and the subject was asked to choose one of three descriptions of the orientations of the encountered shapes: both frontoparallel, both rotated-in-depth, one frontoparallel and the other rotated-in-depth. For this task to be performed successfully, only correct representation of slant-in-depth is needed. Availability of the correct linkages between shape and slant is not required.

\section{Method}

Subjects. The subjects were $\mathbf{4 0}$ undergraduates enrolled in an introductory psychology course at the University of WisconsinMadison. All subjects had normal or corrected-to-normal vision. None of the subjects had participated in the prior experiment.

Stimuli and Apparatus. The shapes and apparatus were the same as those used in Experiment 1.

Procedure. The procedure was the same as that used in Experiment 1 with the exception of the test procedure. After presentation of each pair of shapes, the subjects were asked to evaluate the orientations of the shapes. Three response options were offered: both shapes were in the frontoparallel orientation, both shapes were rotated in depth, or one shape was in frontoparallel orientation and one shape was rotated in depth. For subjects who were exposed to a mixed orientation pair and who responded correctly, an additional inquiry was introduced. These subjects were asked to identify the orientation of the second-presented form.

\section{Results}

Table 2 summarizes the results of Experiment 2. Binomial tests confirmed that the proportion correct exceeded chance for both attentional conditions and all trial types. On trials in which mixed pairs were presented, the probability of correctly identifying the second-presented form given that the trial type was correctly classified was 1.00 for the slant-directed and .882 for the numerositydirected conditions, respectively. Odd-even discrimination was correct on $71.2 \%$ of the trials.

\section{GENERAL DISCUSSION}

The results of this study make two contributions to assessment of our account of the process of perceiving shapeat-a-slant. The results undermine the credibility of the default hypothesis as an interpretation of Epstein and Lovitts's (1985) findings concerning the effects of with-

Table 2

Proportion Correct Under the Attentional Conditions of Experiment 2

\begin{tabular}{lcc}
\hline & \multicolumn{2}{c}{ Attentional Condition } \\
\cline { 2 - 3 } \multicolumn{1}{c}{ Trial Type } & Numerosity-Directed & Slant-Directed \\
\hline Both Frontoparallel & .70 & 1.00 \\
Both Rotated & .70 & 1.00 \\
Frontoparallel-Rotated & 1.00 & 1.00 \\
Rotated-Frontoparallel & .70 & 1.00 \\
Overall & .78 & 1.00 \\
\hline
\end{tabular}

drawal of attention on perceived shapes. Equally important, the results fill a gap in the earlier work by providing positive evidence that slant-in-depth is represented correctly when attention is withdrawn. This demonstration contributes important support for the partial automaticity hypothesis. Additional investigation is needed to determine whether sensitivity to variations of slant-in-depth when attention is not directed to processing slant-in-depth is equivalent to the sensitivity for such variations when attention is directed toward this discrimination. Also required to complete the picture are investigations that segregate the various optical correlates that carry information for slant-in-depth. In the present experiments, several of the recognized optical correlates of perceived slant-in-depth were simultaneously available so that only the general conclusion that slant-indepth can be represented automatically is warranted.

\section{REFERENCES}

Coren, S., \& Porac, C. (1983). The creation and reversal of the Müller-Lyer illusion through attentional manipulation. Perception, 12, 49-54.

EPSTEIN, W. (1973). The process of "taking-into-account" in visual perception. Perception, 2, 267-285.

EPSTEIN, W. (1977). Perceptual constancies in the history of perceptual theory. In. W. Epstein (Ed.), Stability and constancy in visual perception: Mechanisms and processes (pp. 1-22). New York: Wiley.

EPSteIN, W., \& Broota, K. D. (1986). Automatic and attentional components in perception of size-at-a-distance. Perception \& Psychophysics, 40, 256-262.

EPSTEIN, W. , LovirTs, B. E. (1985). Automatic and attentional components in perception of shape-at-a-slant. Joumal of Experimental Psychology: Human Perception \& Performance, 11, 355-366.

GoGEL, W. C. (1967). Cue enhancement as a function of task set. Perception \& Psychophysics, 2, 455-458.

GogeL, W. C. (1978). Size, distance and depth perception. In E. C. Carterette \& M. P. Friedman (Eds.), Handbook of perception. Vol. IX. Perceptual processing (pp. 299-333). New York: Academic Press.

Gogel, W. C., Loomis, J. M., Newman, N. J., Sharkey, T. J. (1985). Agreement between indirect measures of perceived distance. Perception \& Psychophysics, 37, 17-27.

GoGel, W. C., \& TIETZ, J. D. (1976). Adjacency and attention as determiners of perceived motion. Vision Research, 16, 839-845.

Nakayama, K., Silverman, G. H. (1986). Serial and parallel processing of visual feature conjunctions. Nature, 320, 264-265.

Peterson, M. A. (1986). Illusory concomitant motion in ambiguous stereograms: Evidence for nonstimulus contributions to perceptual organization. Joumal of Experimental Psychology: Human Perception \& Performance, 12, 50-60.

Rock, I. (1975). An introduction to perception. New York: Macmillan. Rock, I. (1983). The logic of perception. Boston: Bradford Press.

TsAL, Y. (1984). A Müller-Lyer illusion induced by selective attention. Quarterly Joumal of Experimental Psychology, 36A, 329-333.

Vanderplas, J. M., \& Garvin, E. A. (1959). The association value of nonsense shapes. Journal of Experimental Psychology, 57, 147-154.

\section{NOTE}

1. There is evidence from a study reported by Nakayama and Silverman (1986) employing the visual search paradigm that processing of stereopsis is automatic.

(Manuscript received January 29, 1988; revision accepted for publication July 20,1988 .) 